\title{
Artificial Intelligence for Participatory Health: Applications, Impact, and Future Implications
}

\section{Contribution of the IMIA Participatory Health and Social Media Working Group}

\author{
Kerstin Denecke', Elia Gabarron², Rebecca Grainger ${ }^{3}$, Stathis Th. Konstantinidis ${ }^{4}$, \\ Annie Lau ${ }^{5}$, Octavio Rivera-Romero ${ }^{6}$, Talya Miron-Shatz ${ }^{7}$, Mark Merolli ${ }^{8}$ \\ 1 Bern University of Applied Sciences, Bern, Switzerland \\ 2 Norwegian Centre for E-health Research, University Hospital of North Norway, Norway \\ ${ }^{3}$ University of Otago, Wellington, New Zealand \\ 4 University of Nottingham, Nottingham, UK \\ ${ }^{5}$ Centre for Health Informatics, Australian Institute of Health Innovation, Macquarie University, \\ Australia \\ 6 Universidad de Sevilla, Spain \\ 7 Ono Academic College, Israel, and Winton Centre for Risk and Evidence Communication, \\ Cambridge University, England \\ 8 Swinburne University of Technology, and University of Melbourne, Australia
}

\section{Summary \\ Objective: Artificial intelligence (Al) provides people and professionals working in the field of participatory health informatics an opportunity to derive robust insights from a variety of online sources. The objective of this paper is to identify current state of the art and application areas of $A$ in the context of participatory health. Methods: A search was conducted across seven databases (PubMed, Embase, CINAHL, PsychInfo, ACM Digital Library, IEEExplore, and SCOPUS), covering articles published since 2013. Additionally, clinical trials involving Al in participatory health contexts registered at clinicaltrials.gov were collected and analyzed. \\ Results: Twenty-two articles and 12 trials were selected for review. The most common application of $\mathrm{Al}$ in participatory}

health was the secondary analysis of social media data: self-reported data including patient experiences with healthcare facilities, reports of adverse drug reactions, safety and efficacy concerns about over-the-counter medications, and other perspectives on medications. Other application areas included determining which online forum threads required moderator assistance, identifying users who were likely to drop out from a forum, extracting terms used in an online forum to learn its vocabulary, highlighting contextual information that is missing from online questions and answers, and paraphrasing technical medical terms for consumers.

Conclusions: While Al for supporting participatory health is still in its infancy, there are a number of important research priorities that should be considered for the advancement of the field. Further research evaluating the impact of $A$ in in participatory health informatics on the psychosocial wellbeing of individuals would help in facilitating the wider acceptance of Al into the healthcare ecosystem.

\section{Keywords}

Community-based participatory research; participatory health; artificial intelligence; social media; wearable electronic devices; mobile health

Yearb Med Inform 2019:165-73

http://dx.doi.org/10.1055/s-0039-1677902

\section{Introduction}

Over the last decade, the role of people (or patients) in managing their own health or healthcare has changed dramatically $[1,2]$. A significant paradigm shift has been observed, whereby individuals have moved from being passengers in the health journey to more active, engaged, and empowered partners in the shared decision-making process with their health professionals [2-4]. New ways of creating, developing, and consuming health care goods and services have arisen known as participatory health or the participatory health paradigm. Participatory health informatics involves individuals and representatives of those individuals who would be the main beneficiaries of this technology $[5,6]$. Participatory health focuses on patient-centered initiatives to empower individuals in making more informed health decisions where the individual/patient is at the center of any related healthcare initiative [7]. The term was first coined in 2008 [7]. For example, Eytan provides a definition that suggests participatory health: "Enabled by information, software, and community that we collect or create, we the patients can be effective partners in our own health care, and we the people can participate in reshaping the health 
system itself." [8]. Furst further describes participatory health as "care characterized by the ability to rapidly share, classify, and summarize individual health information with the goals of improving health care systems, experiences, and outcomes via integration of patients and stakeholders." [9]. A number of informatics-related tools can be used in participatory health activities such as: social media, mobile phone health apps, personal health records, health social networks, consumer genomics, and crowdsourced health studies $[4,8,9]$. Each enables the generation, production, or collection of various types of data, reflecting several facets of one's health which includes psychological, physical, social, physiological, or cognitive metrics.

With the reemergence of $\mathrm{AI}$ in healthcare in recent years, there has been growing interest to examine the role of AI within participatory health. The quantified-self movement is illustrative of the participatory health paradigm, where people are able to collect, log, and track indicators to quantify their health, thereby providing insight into their overall health status [10]. However, whilst arguably transformative, technological developments in intelligent computing and analytical methods, namely AI, are providing the opportunity to go even deeper into understanding one's health [11]. This is particularly relevant as people are able to collect increased types, amounts, and quality of data about themselves and their health.

AI techniques and methods provide individuals and professionals working in the field of participatory health informatics with an opportunity to derive robust intelligence from online resources [12]. AI adds another dimension to technologies such as social media, and mobile devices/apps for the purposes of participatory health. For example, through applying AI algorithmic modelling, multiple tasks can be performed such as: replicating or supplementing personalized medical advice from health professionals, diagnosing rare and complex conditions, informing how diseases or outbreaks develop and spread, predicting adverse health events, uncovering how people engage in certain health behaviors or utilize healthcare, and correlating experiences and outcomes from different treatments [13-18]. Whilst the age of AI offers a welcome and innovative way to support participatory health, the field is still maturing, particularly from the perspective of rigorous research evidence.

This paper investigates how AI impacts the field of participatory health and examines which $\mathrm{Al}$ applications exist for participatory health from both patient and clinician perspectives.

\section{Methodology}

\subsection{Search Strategy}

The search strategy was developed in two stages. For the stage 1 search, authors primarily focused on social media interventions within the participatory health context. This is because social media are one of the most widely used tools for individuals within the participatory health context. In the second stage of the search, the focus was related to other, less pervasive, participatory health technologies such as: mobile phones, wearables, and chatbots.

Stage 1: The PICO framework (P-Population, I - Intervention, C - Comparison, O - Outcome) was followed to derive the search strategy [19]. Regarding "Population", we refered to various social media. "Intervention" comprised the multiple AI technologies and methods, and "Outcome" included the impact or resulting participatory health/medicine effects. A comparison intervention was not relevant in this context. Based on this topic specification, we created a list of relevant keywords related to machine learning ( 57 keywords), participatory health (12 keywords), and digital/social media (28 keywords). The search strategy included all these agreed terms. The complete literature search strategy is listed in the supplementay material (Appendix 1). The search was performed on the $10^{\text {th }}$ August 2018, guided by the recommendations of PRISMA guidelines [20]. The PRISMA checklist is available in the supplementary material (Appendix 2). The search covered the following databases: PubMed, Embase, CINAHL, PsychInfo, ACM Digital Library, IEEExplore, and SCOPUS.

Stage 2: Finally, to broaden the scope and include future implementation of AI, we also included clinical trials of AI in participatory health. More specifically, we searched for relevant studies on the trial registration platform clinicaltrials.gov. On October 11, 2018, we searched with the keywords "AI" combined with "mobile phone", "smartphone", "wearable" and the keywords "text-mining", "conversational agent", "chatbot". The complete search strategy for clinical trials is shown in the supplementary material (Appendix 3).

\subsection{Eligibility Criteria and Screening}

We included publications published from 2013 and onwards. These were: 1) primary literature or 2) reviews (scoping reviews, systematic reviews, or meta-analyses); and 3) publications that fulfilled 1) and 2). Publications not meeting these criteria were excluded. The limit of 2013 was used as this captures the last five years, which is the relevant timeframe for rapid technological advances like AI. Through this restriction, the authors wished to represent AI in its present state, whereas the technological capabilities have undergone a change in 2013. References were imported to EndNote $\mathrm{X} 7.8$, and duplicates were removed. The first phase involved two independent reviewers (EG and OR) screening titles and abstracts against inclusion criteria. Full-text of articles meeting inclusion criteria were obtained and carefully examined in a second eligibility round. The agreed articles were included in this review. The search by one reviewer (KD) on clinicaltrials.gov identified twenty-nine trials posted since January 1, 2013; after removing duplicates and checking for relevance, twelve trials were included for the analysis.

\subsection{Review Strategy}

The resulting fulltext paper and trial information were considered by all co-authors to answer the research questions:

- What is the current state of the art in AI techniques applied to data from social media and wearables in the context of participatory health?

- What outcomes and effects are reported in literature on this data using AI?

- Which practical implications (now and 
future) exist from a clinical and patient perspective?

- Do clinicians feel threatened by AI? Do patients feel more empowered?

- What challenges and opportunities are produced by AI? What should the roadmap for future research be?

More specifically, the authors considered several topics in this domain and analyzed the retrieved literature with respect to these topics. These include: AI applications based on data from wearables and social media, and trials using AI in participatory health. From the results, we derived implications from a patient perspective, physician perspective, and from a health systems/community perspective.

\section{Results}

The flowchart of the selection procedure is presented in Figure 1, while Figure 2 shows the trial selection procedure. It can be seen that we included 22 papers and 12 trials in the narrative synthesis.

In this section, we first present $\mathrm{AI}$ applications for participatory health that were described in the literature. Secondly, we present the selected clinical trials.

\subsection{Al Applications Based on Data from Social Media}

Of the 22 papers, 17 (77.3\%) explored AI applications based on data from social media; $\mathrm{n}=13(76.5 \%)$ were secondary analysis of data, and $n=4(23.5 \%)$ were literature reviews. Only one of the 22 papers reviewed technologies that involved interaction with patients or clinicians and examined the capabilities of conversational agents for health-related purposes [21]. The supplementary material (Appendix 4) provides a summary of the papers.

\subsubsection{Platforms and Techniques}

The most common application of AI in participatory health was secondary analysis of data, which was reported and/or reviewed in $n=16$ out of 17 papers [14, 22-35].

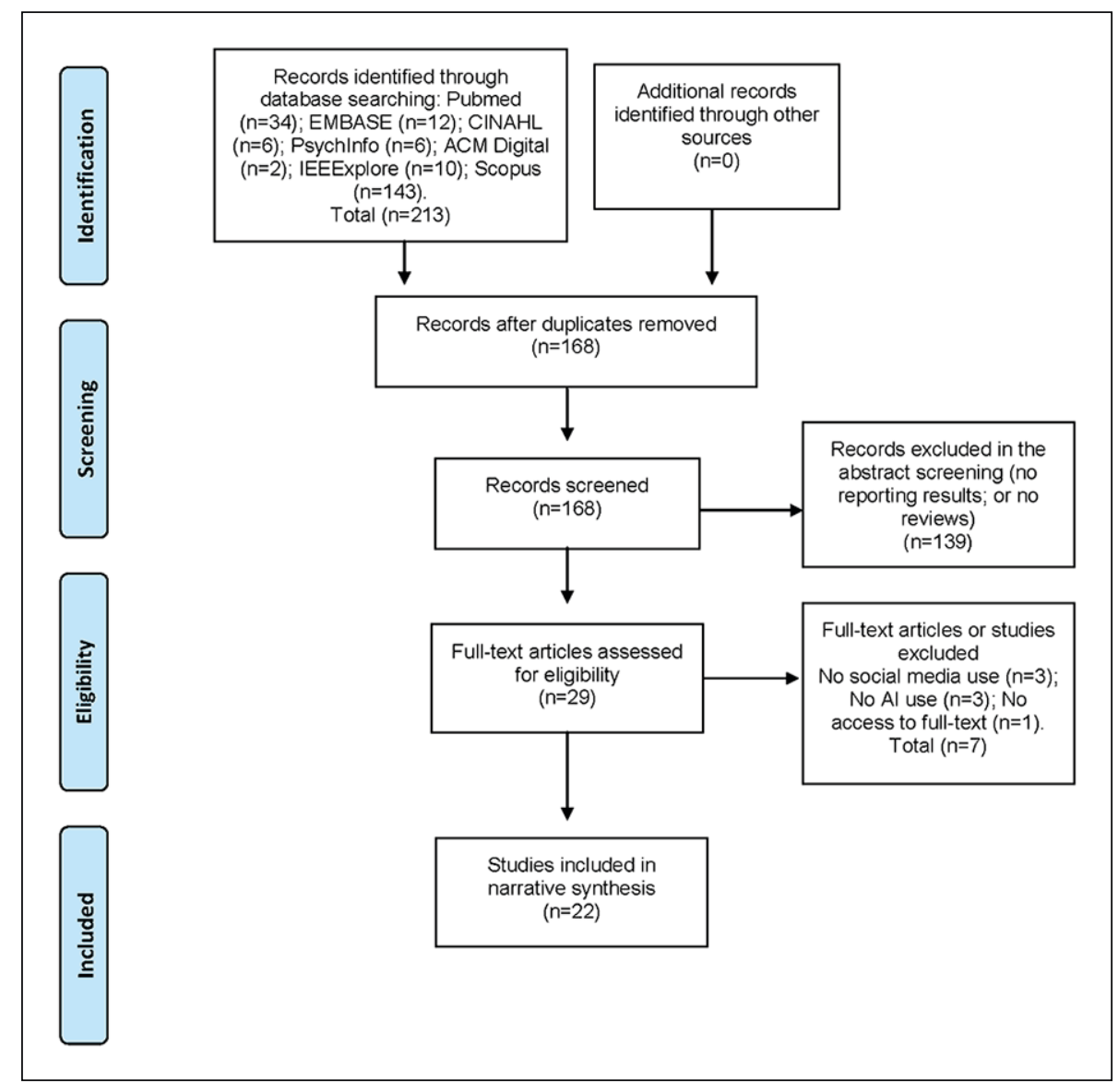

Fig. 1 Flow chart of literature selection resources from bibliographic databases.

These articles primarily reported the use of machine learning techniques to extract health-related data from electronic health records (EHRs) or social media. Secondary data analyses were conducted on general social networks (e.g. Facebook, Twitter, Instagram, Yelp, Amazon, Yahoo Q\&A, Wikipedia, Reddit, LiveJournal), health-specific social networks (e.g. WebMD, RateMD, MedlinePlus, Medlinehelp.org, Breastcancer.org, PatientsLikeMe, DailyStrength, online health forums), and EHR and clinical text (e.g. ShARe, i2b2, THYME).

Most literature ( $\mathrm{n}=9$ out of 17 studies) used text processing techniques, including natural language processing or NLP $(\mathrm{n}=4)[14,24$, $32,33]$, sentiment analysis $(\mathrm{n}=4)[14,27,29$, $30]$, and text classification $(\mathrm{n}=1)$ [28]. Other techniques included classification (3 out of 17 studies) [22, 25, 26], clustering (3 out of 17) [23, 27], conversational agent (1 out of 17 studies) [21], deep learning (1 out of 17 studies) [31], visualization [23, 27], and Latent Dirichlet Allocation [27].

Among the applied text processing and machine learning techniques, sentiment analysis was used to discover safety and efficacy concerns in Amazon reviews for joint and muscle pain relief products [30]. Vocabulary lists used in the analysis consisted of words and phrases that were more indicative of defective products than non-defective products and included non-emotive words that may have been missed by traditional sentiment analysis techniques. Another paper used principal components analysis and logistic regression to identify consumer health expressions (patient terminology) to facilitate adverse drug reactions signals detection among posts that do not use formal medical terminology [34]. A similar study investigated the use of applying word2vec, a 


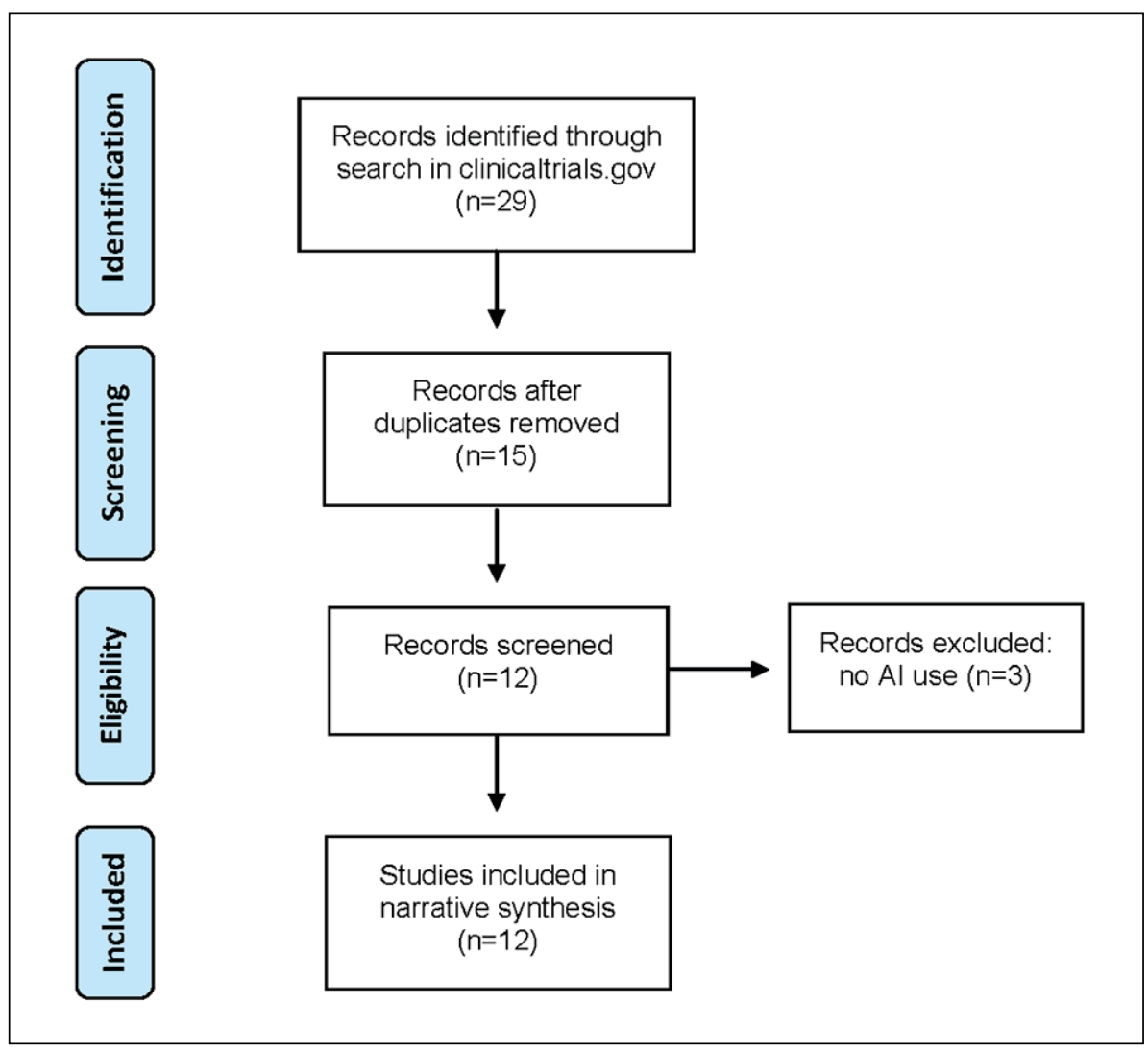

Fig 2 Flow chart of trial selection from clinicaltrials.gov.

word embedding representation, to estimate the rate of adverse drug reactions to psychiatric drugs from social media [31]. Word embedding representations try to predict surrounding words using a central word and vice-versa. This technique was used to better find instances of adverse drug reactions in social media by including colloquial terms such as 'can't sleep', in addition to medical terms such as 'insomnia'. The estimates derived from this approach were found to be similar when compared with official rates from the SIDER database of drug side effects [31].

\subsubsection{Health Conditions and Al Applications}

The most common health condition examined was diabetes, which was investigated in $\mathrm{n}=5$ papers $[22-24,26,35]$. Other conditions included back pain [24], hypertension [24], cancer (including breast cancer) [27, 28, 35], Crohn's disease [29], joint and muscle pain $[29,30]$, mental health [31], lung and respiratory disorders [32], and other chronic conditions [26].

Patients and consumers were the focus of most $(n=14)$ papers [14, 22-32, 17, 35]. Consumers' self-reported data was extracted from social media and included patient experiences at healthcare facilities [14], reports of adverse drug reactions of psychiatric medications [31], safety and efficacy concerns on over-the-counter medications [30], and perspectives on certain medications $[21,29]$. Text-processing techniques were also used to detect disclosure of personal health status information on Twitter [25], identify how consumers express their health concerns $[23,25,35]$, and extract emotional and informational elements in an online post [26]. Other applications included determining which online forum threads required moderator assistance [22], identifying users who were likely to leave an online social network [28], extracting terms used in a question posted online [27], highlighting contextual information that is missing from online questions and answers [27, 32], and paraphrasing technical medical terms for consumers [24]. Facebook data have revealed patients' perspectives and opinions on Crohn's disease and new treatments [29]. One study used conditional Latent Dirichlet Allocation, a weighted generative probabilistic model used to discover latent topics from a collection of documents and clusters of documents with similar distributions of medical terms, to analyze health community discussion threads, specifically in regards to physiological and psychological sentiments for certain conditions. Another study built a corpus called COPE to use social media to understand patient experiences at health care facilities in order to improve the quality of healthcare $[14,27]$. This is crucial, as patients value doctors' personality and demeanor as much as they value their medical care proper [36].

AI has also been used to analyze patients' understanding of medical conditions. One study used Pathfinder networks to analyze the differences in perception of vaccines between physicians and patients, shedding light on patients' level of medical understanding and potentially helping physicians develop better ways of explaining medical issues related to vaccination $[36,37]$.

Both clinicians and consumers were the focus of $n=3$ papers $[21,33,12]$. These included one systematic review of conversational agents [21], and two narrative reviews on NLP techniques in EHR, clinical text, and social media $[12,33]$. These reviews provided commentaries on the state of the art for their respective technology, and highlighted the challenges and the lack of rigorous studies in the field.

Lastly, AI has been used to help healthcare professionals in providing care to patients, both online and in person. One study showed how a low-cost text classification system called Linguistic Inquiry and Word Count (LIWC) assisted moderators in online health communities. The classification determined which posts needed moderators' attention and medical expertise, and which did not. This provided the basis for a system that helps moderators prioritize a selected number of posts that are classified as needing medical 
expertise, rather than making moderators search through thousands of posts in the forum. The system also allowed moderators to change settings depending on how many posts they wanted to see [22]. A second study showed how AI has been used to develop a real-time pneumonia-screening tool to be used in the emergency department that provides care recommendations when pneumonia is suspected from chest $\mathrm{x}$-rays. Emergency departments that employed this tool showed increased adherence with recommendations for treatment of pneumonia $[22,33]$.

\subsubsection{Summary: Al Applications in Participatory Health}

In summary, there are several challenges in this line of research (e.g. working with unlabeled data from unstructured sources), with many studies that remain at the stage of enhancing technical performance and have not yet reached the maturity for user evaluation. Besides the review on conversational agents [21], this paper found neither AI applications that were designed for use by consumers or clinicians in participatory health, nor literature that elicited input from consumers or clinicians for such applications.

The literature to date shows that social media, when analyzed with AI, offers the opportunity to learn about patient's experiences with treatments, to study effects of social media interactions, and many others. None of the included studies reported AI having an effect on clinical outcomes, or an effect of social media on health status. Instead, the literature focused on:

- Content analysis of social media content;

- Term correlation and term usage analysis;

- Opinion or sentiment analysis with respect to treatments;

- Influence of social media interaction with health status.

\subsection{Clinical Trials Studying Al in Participatory Health}

In contrast to published literature aleady reviewed, the 12 identified clinical trials dealt with interventions supported by smartphone applications, broadly grouped in two categories:
- Capturing new kinds of data from clinical trials' subjects using sensors, wearables, and smartphone apps;

- Collecting electronic diaries and patient-reported outcomes using a smartphone app.

A summary of these trials is provided in the supplementary material (Appendix 5). Nine trials tested conversational agents, either embodied (lifelike) or unembodied (unlifelike); one trial assessed the efficiency of a smartphone application, one trial exploited a wearable sensor, and one study the exploitation of Google glasses. Most of the trials randomly assigned people to interventions but were not blinded. The key outcomes were efficiency, impact, and usability of the systems. For example, a randomized control trial evaluating agent technology, i.e. comparing usual care versus usual care plus an AI agent, assessed the impact of the usage of the agent on the quality of life in palliative care [38]. Fitzpatrick et al. performed a trial on an automated conversational agent available as a mobile device smartphone application that provides a cognitive behavioral therapy (CBT). The app allowed patients to immediately process and receive empathy for significant events and removed barriers traditionally limiting treatment access (cost, stigma, health care system navigability, and lack of childcare or transportation) [37]. Another study assessed usability, receptivity, and preliminary cessation when the patient is supported with a conversational agent for smoking cessation [40]. Daily adherence to an embodied conversational agent to use the mobile Kardia heart rhythm monitor has also been evaluated [41].

Two trials evaluated the smartphone intervention IntelliCare. It uses machine learning to tailor treatment for patients suffering from depression and anxiety. The system delivers patient-specific treatment material and motivational messaging via a mobile phone. The purpose of one study was to obtain preliminary information on the feasibility and effectiveness of IntelliCare in improving symptoms of depression and anxiety. The second study is a randomized trial with the application $[42,43]$.

An AI platform on mobile devices has also been used to measure and increase medication adherence in post stroke people with anticoagulation therapy. The AI application visually identified the patient, the medication, and the confirmed ingestion of drugs [44]. Adherence was measured by pill counts and plasma sampling in both groups. Wall et al. studied the effects of an AI tool, running on Google Glass through an Android app, that performs automatic facial expression recognition and delivers social emotion cues to children with autism during social interactions. The system is designed to give participants non-interruptive social cues in real-time and to record social responses that can later be used to help aid behavioral therapy. It studies the effects of the Autism glass intervention [45]. Ally, a mHealth intervention to promote physical activity, is offered in a trial to the customers of a large Swiss health insurance. Interventions include the use of different incentive strategies, a weekly planning intervention and daily message prompts to support self-regulation [46].

In summary, registered clinical trials on AI in participatory health are still limited and are mainly evaluations of conversational agents interacting with patients.

\section{Discussion}

$\mathrm{AI}$ in participatory health informatics can be considered as still in its infancy, as social media and mobile apps were a decade ago. AI applications within participatory health have been assessed in the context of a large variety of health conditions, health promotion activities, and healthcare settings. Furthermore, most evaluations have focused on refining AI methods and techniques, and data on the impact of AI on health outcomes to make meaningful conclusions is still awaited. To date the scholarship of AI in participatory health has been driven by researchers' skills and interests, and in prevalent or challenging healthcare contexts, for example, the high prevalence of diabetes, pain, mental health conditions, or the demands for healthcare experts like neurologists, or cardiologists, whereby healthcare practionners with this expertise are geographically or numerically limited [47, 48]. The next immediately available research data, from a clinical trial identified in this review, will also focus on 
health promotion or adherence to health care interventions and include CBT, smoking cessation, autism, physical activity, and medication adherence $[37,40]$. The very nature of participatory health would suggest that a future research agenda must be informed and directed by people requiring healthcare as a priority, but in partnership with academics and health care providers who understand the context and practicalities of providing robust and meaningful information and knowledge.

\subsection{Implications from the Patient Perspective}

There is potential for AI to make significant improvements to the health of individuals. However, for the field to move forward, one needs to learn the lessons from the previous generation of technologies $[21,49,50]$ to avoid the pitfalls in the design, evaluation, and implementation of patient-facing technologies. These lessons include, but are not limited to: mitigating high participant dropout, avoiding design and implementation not considering patients' context or perspectives, and evaluating findings becoming obsolete by the time they are available.

Patients are often overwhelmed by what they need to do to manage their health. Many of them have busy lives and multiple competing priorities, and are not necessarily familiar with technological developments derived from AI. Asking patients to learn and adopt AI technologies to improve their health and wellbeing may not be something they wish to do, nor a priority for engagement. Patients should be treated as true partners, with research conducted neither 'on' people considered as passive subjects, nor on patient participants only providing data, but research conducted 'with' patients. Patient participants should be considered from initial research development to ensure informaticians are indeed asking the right questions, so that findings provide the right answers to improving patients' lives [51].

Perhaps the challenge of developing patient-facing AI applications lies in human-computer interaction design, not the technology itself [52]. True patient benefit from AI may require the design to be embedded deeply or even invisibly in their daily routine. Without getting a good understanding of the activities patients perform for their health (i.e. patient work), nor how their individual context, digital literacy, or support network affect their health and wellbeing, there is a high risk that patient-facing technology will not be adopted, used, or sustained. One final challenge from this perspective is that of creating, or at least enabling and motivating behavior change using design and psychological mechanisms, as data alone cannot be counted on to bring change and improve health or increase participation [53].

\subsection{Implications from Physician Perspective}

The analysis of social media data with AI has the potential of providing new insights into patient health beliefs and perspectives on their health, healthcare use, and efficacy and adverse effects of drugs and treatments. AI used to detect drug safety and efficacy concerns is attractive as doctors do not always elicit patient medication adherence and concerns about their medication [54]. Furthermore, concerns over side effects and effectiveness are among the main causes of non-adherence $[54,55]$ and they can be revealed by analyzing drug concerns in online health communities.

Sentiment analysis applied to social media data can also be used to discover consumers' feedback on certain medical treatments. This would be helpful to physicians who can gain insight into the prevalence of drug-related events, and how specific drugs work and should be repositionned. Additionally, consumer opinions about new drugs or treatments may be initially found on these sites. By analyzing online health social media data, discovery of drug-drug interactions could change prescription practice, identify contraindications to combination use, or even lead to timely drug withdrawal from the market [56]. This research can be used by the healthcare and pharmaceutical industry to reduce risks for consumers [30, 56]. Furthermore, it could potentially identify unexpected or infrequent drug reactions that would otherwise have been missed. The systematic mining of social media data can provide valuable information for physicians about their patients. For example, one tweet reads "my mom makes having cancer look easy", while another reads such as "I have seasonal allergy". These tweets can help physicians learn about their patients and gain insight into patients' family medical history that may otherwise be missing from electronic medical records [25, 33].

Beyond this, data collected by wearables and through smartphone applications can provide insights into the health of patients. In particular, changes in health parameters become more obvious. However, what needs to be avoided is that physicians are overwhelmed with additional data to be assessed during patient encounters. AI can help in summarizing data and in discovering patterns in data being presented and assessed by physicians.

In summary, these findings show the promise for utilising $\mathrm{AI}$ at the service of physicians, who can use it to gain insight into medications and conditions. All this, often without the patient's knowledge, or active participation.

\subsection{Implications from a Health Systems/Community Perspective}

Information communication technology (ICT) has long been heralded for its potential to improve health systems. The power of larger and more readily available data sets to improve healthcare efficiency and effectiveness, as well as decision-making, has long been debated. However, when combined with advances in cognitive processing and analytics, such as those afforded through AI, the potential for health ICT to generate health system level benefits increases exponentially. According to Accenture, growth in the AI health market is tipped to surpass well-over US\$ 6 billion by 2021 [57].

The application of AI to participatory health informatics has started and focused on the secondary analysis of data produced within participatory health activity (as illustrated by the findings of this review with $n=16$ out of the 17 selected papers). This may be due in part because permissions to obtain access to social media and networking data is easier than obtaining data from 
custom databases related to mobile devices, or obtaining access to national health system electronic records. To date $\mathrm{AI}$ in participatory health has focused on text-related data. That should not create a stereotype that participatory health informatics focuses on text data only, since there are examples that use EHR data. In the future it can encompass the use of physiological data used from wearable devices $[58,59]$ and clinical trials (as also identified in this review) are employing devices (sensors, wearables, and smartphone apps) to capture data. To increase knowledge about impact of AI applications on participatory health and health outcomes, research frameworks and communities will need to work through the ethical and practical privacy issues of research using healthcare data, such as medical images [60], or biological data [61]. This is since transformative insights are most likely to be achieved when conventional health data (EHR, imaging, prescribing records) are combined with patient-generated data, e.g. both experiential reporting and physiological data. These issues need to be urgently addressed by health systems, regulators, and society [62].

AI can also influence positive changes in participatory health informatics by providing insights to improve health systems functioning for individuals and communities. AI applications offer organizations in the health system insights and solutions to offsest rising financial pressures, increasing costs of healthcare, ageing populations, increasing incidences of complex chronic diseases, shortages of skilled professionals, and rising consumerism and digital patient empowerment $[18,57,63]$. If these improve the care experience, either directly or indirectly by freeing up resources, this will have a positive impact on participatory health.

Consequently, as the field matures, AI has the potential to assist health systems globally through: improved quality of healthcare, improved workflows, error reduction, better accuracy of information, enhanced safety, better access, greater user (patient)-centeredness, more personalized and preventative healthcare, and ultimately cost savings [18, 57]. Furthermore, and highly relevant to the domain of participatory health informatics, is that among the top 10 application areas for AI proposed by Accenture, at least four arguably have participatory health connotations, including: robotics, virtual health professionals/assistants, connected devices, and recruitment of participants into clinical trials [64]. Some of them have already started to be explored, with large areas for future research.

It has been already noted that $\mathrm{AI}$ offers tremendous potential to support and progress the diagnosis of key conditions on the World Health Organisation's health burden list [9], for example, cancer, neurological and cardiac conditions [11]. In these conditions, the major application of AI centers on diagnosis and relative risk prediction. However, before AI can play a meaningful role in supporting models of participatory health in the community, further work and research is needed on its utility to support treatment, and emerging models of care [11, 66]. Despite this, AI techniques are already being leveraged for treatment purposes, for example to support movement of prostheses in stroke and spinal cord injury [11].

\section{Conclusions and Roadmap for Future Research}

The authors are cognizant of the impact and growing interest of $\mathrm{AI}$ in the field of participatory health, increased research funds being made available, and advances in the analysis, classification, and prediction of participatory health related data over the past five years. Nevertheless, many challenges remain to be addressed before $\mathrm{AI}$ is able to have a measurable impact on participatory health. More coordinated efforts between local, regional, and international research networks to develop an actionable and realistic research agenda for AI research in participatory health is needed. Such a roadmap for research should be able to separate the dissonance between the hype and the reality of AI in participatory health informatics, and be actionable within the realities of the contemporary global healthcare context.

There are numbers of research challenges as well as opportunities for $\mathrm{AI}$ in participatory health that need to be considered. The introduction of AI technologies for partici- patory health will provide health consumers access to a global health network including of healthcare services and access to other like-minded health consumers. Some of the immediate research challenges to be considered are where AI for participatory health best fits within the global healthcare spectrum in relation to preventive health, home care, primary care, secondary, and tertiary care. In particular, the focus should perhaps be put on developed nations that have a larger proportion of elderly individuals, versus developing countries with a larger proportion of young adults and adolescents. Also, AI-related technologies empower health consumers and may reduce health consumer reliance on medical advice which makes the impacts of $\mathrm{AI}$ on the patient-doctor relationship difficult to predict. Furthermore, technological advances in AI are increasing dramatically and evaluating AI impact on consumer engagement and general patient well-being remains to be investigated. An Ernst and Young 2017 research report indicates that for participatory health to become embedded within the healthcare system, three important elements must be considered: 1) capitalization on the advances of technology; 2) empowering platforms that combine technologies, people, and processes; and 3) cultural shifts that enable the system transition towards an ecosystem [67].

Considering all these factors, there are a number of key and important research priorities that should be considered for the advancement of AI research in participatory health (Figure 3). Since healthcare ecosystems are slow to change, AI technology will continue to advance and participatory health informatics will need to adapt to the technological advancements of AI, as more demands from health consumers and the healthcare industry place pressure on the overall healthcare ecosystem to use, apply, and accept AI technology. More work to understand the impact of AI on participatory health is needed, as explained and illustrated below in the suggested research priority areas for AI in participatory health:

1. Evaluation - Research evaluating the impacts of AI on participatory health is needed. In particular research around medical errors, technology acceptance, 
Denecke et al.

healthcare costs, quality of care, and impacts on decision making;

2. Patient-Clinician Relationship Research evaluating the impacts of AI on patient-clinician relationships is needed as the autonomy of physicians decreases due to more empowered patients, whereas empowerment and engagement of consumers through AI technology increase;

3. Healthcare Readiness - Healthcare organizations are slow to change and research into the readiness of healthcare organizations to accept AI is needed;

4. Technology Adoption - Research to understand how healthcare professionals and consumers, of all ages, are ready to accept $\mathrm{AI}$ as a complementary or alternative type of care;

5. Psychosocial Wellbeing - Researching the psychosocial impacts of participatory health, especially, on health consumers is needed as more interactions occur with an artificial agent vs. human interaction;

6. Privacy and Security - Research on sharing health information with AI technology and the impacts on patient privacy and security should be addressed;

7. Technology Advancement - As AI technology is advancing at a rapid pace, research on the types and relevance of AI technology for participatory health is required.

\section{References}

1. Finset A. Patient Participation, Engagement and Activation: Increased emphasis on the role of patients in healthcare. Patient Educ Couns 2017;100(7):1245-46.

2. deBronkart D. From patient centred to people powered: autonomy on the rise. BMJ 2015;350:h148.

3. Millenson ML. When "patient centred" is no longer enough: the challenge of collaborative health: an essay by Michael L Millenson. BMJ 2017;358:j3048.

4. Coughlin S, Roberts D, O'Neill K, Brooks P. Looking to tomorrow's healthcare today: a participatory health perspective. Intern Med J 2018;48(1):92-6.

5. International Collaboration for Participatory Health Research (ICPHR). Position Paper 1: What is Participatory Health Research? 2013. Available from: http://www.icphr.org/ uploads/2/0/3/9/20399575/ichpr_position_paper_1_defintion_-_version_may_2013.pdf

6. Society for Participatory Medicine. What is Participatory Medicine? 2017. Available from: https://participatorymedicine.org/what-is-participatory-medicine/.

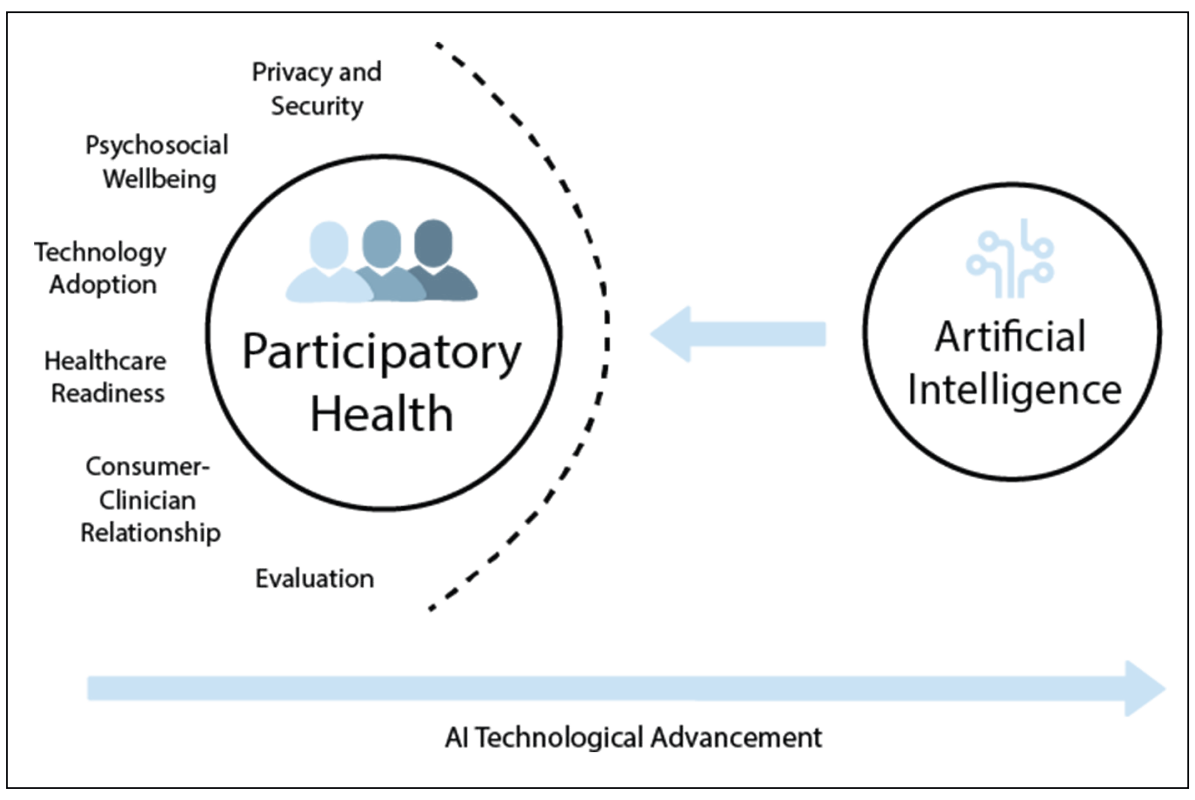

Fig. 3 Research priority areas for participatory health in the age of Al.

7. Swan M. Health 2050: The Realization of Personalized Medicine through Crowdsourcing, the Quantified Self, and the Participatory Biocitizen. J Pers Med 2012;2(3):93-118.

8. Eytan T. eHealth. Patient empowerment. The Health 2.0 Definition: Not Just the Latest, the Greatest! [Cited: 3 March 2019] Available: http:// www.tedeytan.com/tag/definition.

9. Furst I. Wait Time and Delayed Care. New Health 2.0 Definition [Cited 03 March 2019]. Available: http://waittimes.blogspot.com/2008/06/newhealth-20-definition.html.

10. Almalki M, Gray K, Sanchez FM. The use of self-quantification systems for personal health information: big data management activities and prospects. Health Inf Sci Syst 2015;3:S1.

11. Jiang F, Jiang Y, Zhi H, Dong Y, Li H, Ma S, et al. Artificial intelligence in healthcare: past, present and future. Stroke Vasc Neurol 2017;2:230.

12. Gonzalez-Hernandez G, Sarker A, O'Connor K, Savova G. Capturing the Patient's Perspective: a Review of Advances in Natural Language Processing of Health-Related Text. Yearb Med Inform 2017;26(1):214-27.

13. Charles-Smith LE, Reynolds TL, Cameron MA, Conway M, Lau EHY, Olsen JM, et al. Using Social Media for Actionable Disease Surveillance and Outbreak Management: A Systematic Literature Review. PLoS One 2015;10:e0139701.

14. Rastegar-Mojarad M, Ye Z, Wall D, Murali N, Lin S. Collecting and Analyzing Patient Experiences of Health Care From Social Media. JMIR Res Protoc 2015;4:e78.

15. Lu Y, Wu Y, Liu J, Li J, Zhang P. Understanding Health Care Social Media Use From Different Stakeholder Perspectives: A Content Analysis of an Online Health Community. J Med Internet Res 2017;19(4):e109.

16. Yang FC, Lee AJ, Kuo SC. Mining Health Social Media with Sentiment Analysis. J Med Syst 2016;40(11):236.

17. Staccini P, Fernandez-Luque L. Secondary Use of Recorded or Self-expressed Personal Data: Consumer Health Informatics and Education in the Era of Social Media and Health Apps. Yearb Med Inform 2017;26(1):172-77.

18. Roberts D, Marion G, Coughlin S, Brooks P. Health reimagined: a new participatory health paradigm. Ernst \& Young, Australia, 2016 [cited 29 Sep 2018]. Available: https://www.ey.com/ Publication/vwLUAssets/EY_-_Health_reimagined:_a_new_participatory_health_paradigm $/ \$$ FILE/ey-health-reimagined-2016.pdf.

19. Thompson M, Tiwari A, Fu R, Moe E, Buckley DI. A Framework To Facilitate the Use of Systematic Reviews and Meta-Analyses in the Design of Primary Research Studies. Rockville (MD): Agency for Healthcare Research and Quality (US); 2012.

20. Moher D, Liberati A, Tetzlaff J, Altman DG. Preferred Reporting Items for Systematic Reviews and Meta-Analyses: The PRISMA Statement. J Clin Epidemiol 2009;62:1006-12.

21. Laranjo L, Dunn AG., Tong HL, Kocaballi AB, Chen J, Bashir R, et al. Conversational agents in healthcare: a systematic review. J Am Med Inform Assoc 2018;25:1248-58.

22. Huh J, Yetisgen-Yildiz M, Pratt W. Text classification for assisting moderators in online health communities. J Biomed Inform 2013;46:998-1005.

23. Zhang J, Zhao Y, Dimitroff A. A study on health care consumers' diabetes term usage across identified categories. Aslib Journal of Information Management 2014;66:443-63.

24. Grabar N, Hamon T. Automatic Extraction of Layman Names for Technical Medical Terms. 
2014 IEEE International Conference on Healthcare Informatics; 2014. doi:10.1109/ichi.2014.49.

25. Yin Z, Fabbri D, Rosenbloom ST, Malin B. A Scalable Framework to Detect Personal Health Mentions on Twitter. J Med Internet Res 2015;17:e138.

26. Deetjen U, Powell JA. Informational and emotional elements in online support groups: a Bayesian approach to large-scale content analysis. J Am Med Inform Assoc 2016;23:508-13.

27. Yang F-C, Lee AJT, Kuo S-C. Mining Health Social Media with Sentiment Analysis. J Med Syst 2016;40:236.

28. Wang X, Zhao K, Street N. Analyzing and Predicting User Participations in Online Health Communities: A Social Support Perspective. J Med Internet Res 2017;19:e130.

29. Roccetti M, Marfia G, Salomoni P, Prandi C, Zagari RM, Gningaye Kengni FL, et al. Attitudes of Crohn's Disease Patients: Infodemiology Case Study and Sentiment Analysis of Facebook and Twitter Posts. JMIR Public Health Surveill 2017:3:e51.

30. Adams DZ, Gruss R, Abrahams AS. Automated discovery of safety and efficacy concerns for joint \& muscle pain relief treatments from online reviews. Int J Med Inform 2017;100:108-20.

31. Nguyen T, Larsen ME, O'Dea B, Phung D, Venkatesh S, Christensen H. Estimation of the prevalence of adverse drug reactions from social media. Int J Med Inform 2017;102:130-7.

32. Eletriby MR, Reynolds TL, Jain R, Zheng K. Investigating named entity recognition of contextual information in online consumer health text. 2017 Eighth International Conference on Intelligent Computing and Information Systems (ICICIS); 2017. doi:10.1109/intelcis.2017.8260078.

33. Demner-Fushman D, Elhadad N. Aspiring to Unintended Consequences of Natural Language Processing: A Review of Recent Developments in Clinical and Consumer-Generated Text Processing. Yearb Med Inform 2016; 224-33.

34. Jiang L, Yang CC, Li J. Discovering Consumer Health Expressions from Consumer-Contributed Content. Lecture Notes in Computer Science; 2013. p. 164-74.

35. He Z, Chen Z, Oh S, Hou J, Bian J. Enriching consumer health vocabulary through mining a social Q\&A site: A similarity-based approach. J Biomed Inform 2017;69:75-85.

36. Miron-Shatz T, Becker S, Zaromb F, Mertens A, Tsafrir A. "A Phenomenal Person and Doctor": Thank You Letters to Medical Care Providers. Interact J Med Res 2017;6:e22.

37. Amith M, Cunningham R, Savas LS, Boom J, Schvaneveldt R, Tao C, et al. Using Pathfinder networks to discover alignment between expert and consumer conceptual knowledge from online vaccine content. J Biomed Inform 2017:74:33-45.

38. Paasche-Orlow M. Conversational Agents to Improve Quality of Life in Palliative Care (ECA-PAL). https://clinicaltrials.gov/ct2/show/ NCT02750865. 26 April 2016.

39. Fitzpatrick KK, Darcy A, Vierhile M. Delivering Cognitive Behavior Therapy to Young Adults With Symptoms of Depression and Anxiety Using a Fully Automated Conversational Agent (Woebot): A Randomized Controlled Trial. JMIR Ment Health 2017;4:e19.
40. Sullivan B. Pilot Trial of the First Conversational Agent for Smoking Cessation (QuitBot); 2018. https://clinicaltrials.gov/ct2/show/NCT03585231.

41. Magnani, J.W. Pilot Atrial Fibrillation Information Technology Trial; 2017. https://clinicaltrials.gov/ ct2/show/NCT03093558.

42. Mohr, D. IntelliCare: Artificial Intelligence in a Mobile Intervention for Depression and Anxiety (AIM); 2014. https://clinicaltrials.gov/ct2/show/ NCT02176226.

43. Mohr DC, Tomasino KN, Lattie EG, Palac HL, Kwasny MJ, Weingardt K, et al. IntelliCare: An Eclectic, Skills-Based App Suite for the Treatment of Depression and Anxiety. J Med Internet Res 2017:19:e10.

44. Labovitz DL, Shafner L, Reyes Gil M, Virmani D, Hanina A. Using Artificial Intelligence to Reduce the Risk of Nonadherence in Patients on Anticoagulation Therapy. Stroke 2017;48:1416-9.

45. Wall DP. Examining the Efficacy of a Mobile Therapy for Children With Autism Spectrum Disorder; 2018. https://clinicaltrials.gov/ct2/show/ NCT03569176.

46. Kowatsch, T. Can a Smartphone App That Includes a Chatbot-based Coaching and Incentives Increase Physical Activity in Healthy Adults? 2017. https:// clinicaltrials.gov/ct2/show/NCT03384550.

47. Farina D, Vujaklija I, Sartori M, Kapelner T, Negro F, Jiang N, et al. Man/machine interface based on the discharge timings of spinal motor neurons after targeted muscle reinnervation. Nature Biomedical Engineering, 2017;1: Article number:0025.

48. Bouton CE, Shaikhouni A, Annetta NV, Bockbrader MA, Friedenberg DA, Nielson DM, et al. Restoring cortical control of functional movement in a human with quadriplegia. Nature 2016;533: 247-50.

49. Lau AY, Arguel A, Dennis S, Liaw S-T, Coiera E. "Why Didn't it Work?" Lessons From a Randomized Controlled Trial of a Web-based Personally Controlled Health Management System for Adults with Asthma. J Med Internet Res 2015;17:e283.

50. Lau AY, Piper K, Bokor D, Martin P, Lau VS, Coiera E. Challenges During Implementation of a Patient-Facing Mobile App for Surgical Rehabilitation: Feasibility Study. JMIR Hum Factors 2017;4:e31.

51. International Collaboration for Participatory Health Research. What is Participatory Health Research? Version: Mai 2013. Berlin: International Collaboration for Participatory. In: Position Paper [Internet]. 2013 [cited 10 Mar 2018]. Available: http://www.icphr.org/uploads/2/0/3/9/20399575/ ichpr_position_paper_1_defintion_-_version_ may_2013.pdf.

52. What We Get Wrong About Artificial Intelligence. In: IOTForALL [Internet]. 16 Nov 2017 [cited 10 Mar 2018]. Available: https:/www.iotforall.com/ human-computer-interaction-design-ai/.

53. Miron-Shatz T. Beyond the Pill: A novel Take on Digital Health can Increase its Effectiveness. BMJ Clinical Evidence Blog October 7th, 2016.

54. Brown MT, Sinsky CA. Medication Adherence: We Didn't Ask and They Didn't Tell. Fam Pract Manag 2013;20:25-30.

55. McHorney CA. The Adherence Estimator: a brief, proximal screener for patient propensity to adhere to prescription medications for chronic disease. Curr Med Res Opin 2009;25:215-38.
56. Jiang L. Recommendations in online health communities with heterogeneous information network mining (Order No. 10131821). Available from ProQuest Dissertations \& Theses A\&I., editor. PhD, Drexel University; 2016.

57. Collier M, Fu R, Yin L, Christiansen P. Why artificial intelligence is the future of growth. Accenture; 2017. [cited 29 Sep 2018]. Available: https:// www.accenture.com/t20170418T023052Z _ w / / au-en/_acnmedia/PDF-49/Accenture-Health-Artificial-Intelligence.pdf\#zoom $=50$.

58. Konstantinidis EI, Frantzidis CA, Papadelis C, Pappas C, Bamidis PD. WADEDA: A Wearable Affective Device with On-Chip Signal Processing Capabilities for Measuring ElectroDermal Activity. In: Bamidis PD, Pallikarakis N, editors. XII Mediterranean Conference on Medical and Biological Engineering and Computing 2010. IFMBE Proceedings, 2010, vol 29. Berlin, Heidelberg: Springer; 2010.

59. Bellazzi R. Telemedicine and Diabetes Management: Current Challenges and Future Research Directions. J Diabetes Sci Technol 2008;2(1):98-104.

60. Gillies RJ, Kinahan PE, Hricak H. Radiomics: Images Are More than Pictures, They Are Data. Radiology 2015;278(2):563-77.

61. Li C, Liang G, Yao W, Sui J, Shen X, Zhang Y, et al. Integrated analysis of long non-coding RNA competing interactions reveals the potential role in progression of human gastric cancer. Int J Oncol 2016;48:1965-76

62. Ballantyne A. Where is the human in the data? A guide to ethical data use. GigaScience 2018;7(7).

63. Fineberg HV. Shattuck Lecture. A successful and sustainable health system--how to get there from here. N Engl J Med 2012 Mar 15;366(11):1020-7.

64. Litwin A. Using Artificial Intelligence To Monitor Medication Adherence in Opioid Replacement Therapy; 2018. https://clinicaltrials.gov/ct2/show/ NCT02243670.

65. Mitchell S. Treating Comorbid Depression During Care Transitions Using Relational Agents; 2018. https://clinicaltrials.gov/ct2/show/NCT02845102.

66. Dobson R, Whittaker R, Dale LP, Maddison $R$. The effectiveness of text message-based self-management interventions for poorly-controlled diabetes: A systematic review. Digit Health 2017;3:205520761774031.

67. Ernst and Young. Healthcare: the cross-currents of convergence deliver participatory health. In: Ey.com [Internet]; 2017 [cited 10 Jan 2018]. Available: https://www.ey.com/Publication/vwLUAssets/EY-healthcare-the-cross-currents-of-convergence-deliver-participatory-health/\$FILE/ EY-healthcare-the-cross-currents-of-convergence-deliver-participatory-health.pdf.

68. Wong J. A Study Evaluating Sleep, Stress and Infant Nutrition Using a Chatbot; 2018. https:// clinicaltrials.gov/ct2/show/NCT03630679.

\section{Correspondence to:}

Kerstin Denecke

Bern University of Applied Sciences

Quellgasse 21

2501 Biel, Switzerland

Tel.: +41323216794

E-mail: kerstin.denecke@bfh.ch 